\title{
Clinical and Therapeutic Profile of Undifferentiated Nasopharyngeal Carcinoma in Children and Young Adults: A Retrospective Study
}

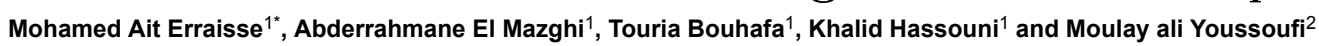

${ }^{1}$ Department of Radiotherapy, Hassan II University Hospital, Fez, Morocco

${ }^{2}$ Medical physics unit, Hassan II University Hospital, Fez, Morocco

*Corresponding author: Mohamed Ait Erraisse, Department of Radiotherapy, Hassan II University Hospital, Fez, Morocco, E-mail: aiterraissemohamed@gmail.com

Received date: August 10, 2018; Accepted date: September 12, 2018; Published date: September 20, 2018

Copyright: (C2018 Erraisse MA, et al. This is an open-access article distributed under the terms of the Creative Commons Attribution License, which permits unrestricted use, distribution, and reproduction in any medium, provided the original author and source are credited.

\begin{abstract}
Objective: The aim of our work was to analyze the epidemiological, clinical and therapeutic aspects of nasopharyngeal cancer in children and young adults under 20 years of age.

Patients and Methods: This was a retrospective study of 20 patients diagnosed with undifferentiated nasopharyngeal carcinoma between June 2012 and October 2016 and treated at the radiotherapy department of Hassan II University Hospital in Fez, Morocco.

Results: The average age of patients was 13.5 years. The most affected age group was 10 to 15 (14 patients). The male/female ratio was $1,8: 1$. The diagnosis is often late with presenting symptomatology dominated by cervical lymphadenopathy in 95\% of cases. Fifteen patients were classified T3-T4 and 14 patients were N2-N3 with one bone metastasis. All patients received neoadjuvant chemotherapy followed by concomitant radiochemotherapy. Three patients were lost to follow-up. The acute toxicity was radiation induced mucositis $(100 \%)$, radiodermatitis $(60 \%)$, xerostomia $(55 \%)$, hearing impairement $(44 \%)$ and trismus $(33 \%)$. There was good locoregional control in $55 \%$, loco-regional recurrence in $10 \%$, and distant metastases in $15 \%$. The overall survival was $85 \%$ and relapsefree survival was $50 \%$. Conclusion: Improving the prognosis of this cancer requires early diagnosis and more accessible and developed medical infrastructures. Recent advances in intensity modulated radiation therapy should be the main treatment technique for all our patients.
\end{abstract}

Keywords: Nasopharynx; Children; Radiotherapy; Chemotherapy; Prognosis

\section{Introduction}

Childhood nasopharyngeal cancer in young adults are common in countries around the Mediterranean where it accounts for $5 \%$ to $10 \%$ of all pediatric tumors and $18 \%$ of nasopharyngeal carcinomas $[1,2]$ The current management guidelines are similar to those of adults without specific recommendations to this age group. Radiation therapy remains the main treatment modality with concomitant chemotherapy. The squeal of treatment in children and young adults remain the main challenge [3]. Although the stages of the disease are usually advanced in children and young adults, the prognosis remains better compared to adults $[4,5]$. In this study, we will highlight the clinical and therapeutic aspects of this cancer in 20 children and young adults.

\section{Patients and Methods}

This was a retrospective study of 20 patients aged $<20$ years who had histologically proven nasopharyngeal carcinoma either by nasopharynx biopsy or cervical lymph node biopsy. The study was carried out from June 2012 to October 2016 at the radiotherapy department of Hassan II University Hospital in Fez, Morocco. The patients had a clinical examination, a biopsy of the nasopharyngeal mass or cervical lymphadenopathy, as well as intr-oral assessment. The loco-regional extension assessment was made with CT-scan (15 patients) and/or cervicofacial MRI (5 patients). The distance assessment was done with chest X-ray, abdominal ultrasound and thoracoabdominopelvic CT scan. All patients underwent bone scintigraphy. At the end of this assessment, the patients were classified according to the AJCC $7^{\text {th }}$ Edition.

\section{Treatment sequence}

The patients had external beam radiotherapy. The protocol includes induction chemotherapy followed by concomitant radio chemotherapy.

\section{Radiotherapy}

Radiotherapy was delivered using 3D conformal radiotherapy (3DCRT) technique in 15 patients. The prescribed dose was 70 Gy on tumor and positive lymph nodes and 50 Gy in low risk areas. The Photon beamswere $6 \mathrm{MeV}$ and the electron beams were 6,9 or $12 \mathrm{MeV}$.

After contouring the target volumes (Gross tumor volume (GTV), Clinical target volume (CTV), Planning target volume (PTV)) and organs at risk. A first course of radiotherapy was delivered to the primary tumor areas as well as the microscopic extensions (depending on imaging) and uninvolved nodes up to a dose of $40 \mathrm{~Gy}$. A boost on the nasopharynx up to a total of 7 Gy was delivered. The neck was treated using electron beams up to a dose of 50 Gy or 70 Gy depending the extent of nodal involvement. In No patients, a five-beam technique was used to allow us to give $50 \mathrm{~Gy}$ to the neck and nasopharynx without electron beam (Figure 1). 
Citation: Erraisse MA, Mazghi AE, Bouhafa T, Hassouni K, Youssoufi MA (2018) Clinical and Therapeutic Profile of Undifferentiated Nasopharyngeal Carcinoma in Children and Young Adults: A Retrospective Study. J Nucl Med Radiat Ther 9: 377. doi: $10.4172 / 2155-9619.1000377$

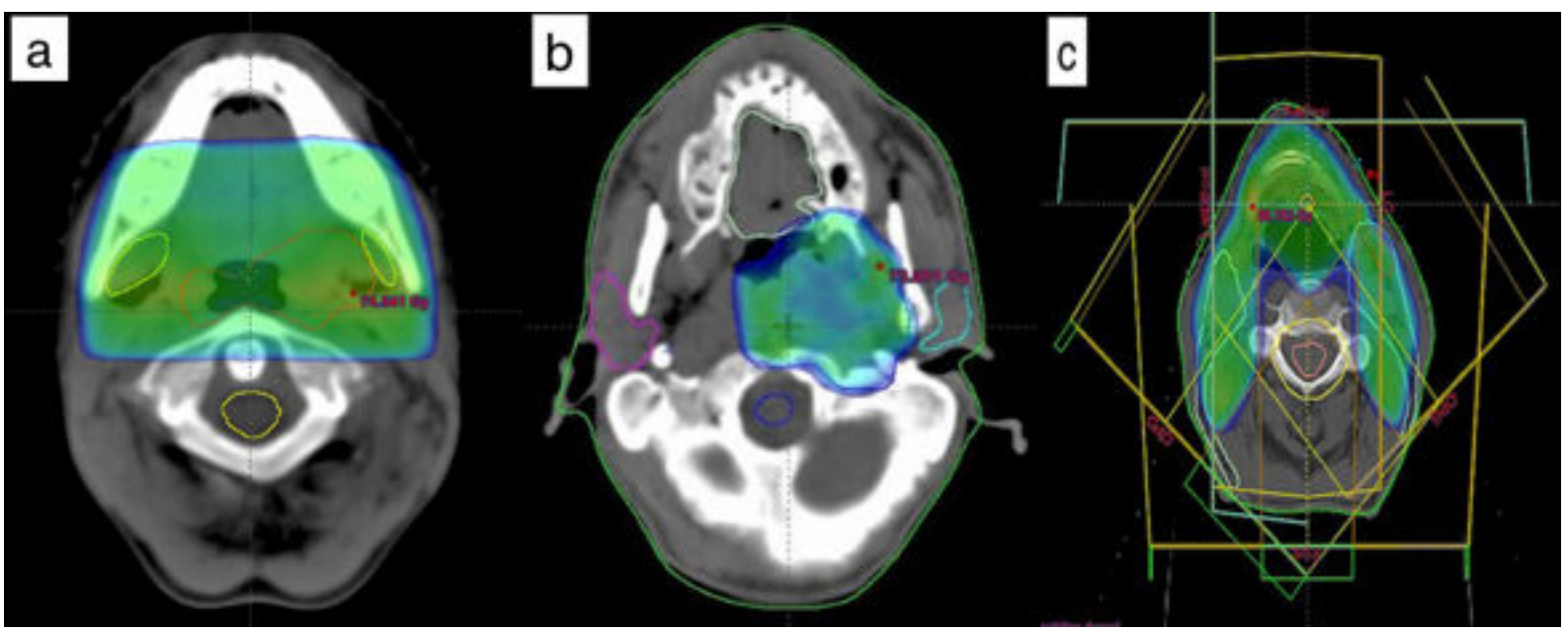

Figure 1: (a): Dosimetry example in 3DCRT showing the isodose line of 70 Gy. Parotids (in yellow) are not spared. (b): Dosimetry in IMRT showing the better conformity to the volume with sparing of parotids and brainstem. (c): 5 field's technique isodose of 50 Gy with respect of the spinal cord.

Intensity modulated radiation therapy (IMRT) technique was used in 5 patients. The prescription was made according to the principle of the simultaneous integrated boost $(\mathrm{SiB})$ therapy: A gross tumor volume (tumor+lymphadenopathy) received 70 Gy at 2 Gy per fraction, then the volume at high risk of invasion received $63 \mathrm{~Gy}$ at 1.8 Gy per fraction while the low risk volume received a dose of $56 \mathrm{~Gy}$ to $1.6 \mathrm{~Gy}$ per fraction. In all cases, the contouring was done with respect to pre-chemotherapy tumor volume.

\section{Chemotherapy}

Induction chemotherapy consisted of Doxorubicin-Cisplatin in 4 patients over 16 years old and Bleomycin-Epirubicin-Cisplatin in the rest. Chemotherapy was administered every 21 days for a total of 3 cycles. The concomitant chemotherapy was made of weekly cisplatin at a dose of $30 \mathrm{mg} / \mathrm{m}^{2}$.

\section{Treatment monitoring}

Complete remission is defined as total disappearance of any known lesion. Partial remission is defined as decrease of more than $50 \%$.

Patients were seen weekly during chemo radiotherapy. After the end of treatment, they are seen every three months for two years, then every six months until the fifth year and then annually.

\section{Results}

The average annual frequency was 4 new cases per year. The mean age at diagnosis was 13.5 years (from 10 years to 18 years). The peak frequency was between 10 and 15 years old. There were 13 boys (65\%) and 7 girls (35\%) giving M/F sex ratio of 1.8:1. Lymphadenopathy was present in 19 cases (95\%), an otological syndrome in 15 patients $(75 \%)$, and a rhinological and neurological syndrome in 14 patients $(70 \%)$. The average time to consultation was 7 months. The diagnosis was confirmed by a nasopharynx biopsy in 15 patients (75\%), and a lymph node biopsy in 5 patients (25\%). The tumor was classified as T3 and T4 in $30 \%$ and $45 \%$ of patients respectively. The lymph node involvement was classified as N2 and N3 in 55\% and $15 \%$ of patients respectively. For the distant extension assessment, we found a patient with a bone metastasis on bone scan. EBV (Epstein Barr Virus) serology was performed in one patient and was positive (Table 1).

\begin{tabular}{|c|c|}
\hline Patients Characteristics & Number (\%) \\
\hline \multicolumn{2}{|l|}{ Sex } \\
\hline Male & $13(65)$ \\
\hline Female & $7(35)$ \\
\hline \multicolumn{2}{|l|}{ Age } \\
\hline$\geq 16$ & $4(20)$ \\
\hline$<16$ & $16(80)$ \\
\hline \multicolumn{2}{|l|}{ Symptoms } \\
\hline Lymphadenopathy & $19(95)$ \\
\hline Nasal obstruction & $6(30)$ \\
\hline Nasal bleeding & $10(50)$ \\
\hline Headache & $13(65)$ \\
\hline Trismus & $3(15)$ \\
\hline \multicolumn{2}{|l|}{ Pathology } \\
\hline UCNT & $20(100)$ \\
\hline Induction Chemotherapy & $20(100)$ \\
\hline T3/T4 & $15(75)$ \\
\hline N2/N3 & $14(70)$ \\
\hline
\end{tabular}


Citation: Erraisse MA, Mazghi AE, Bouhafa T, Hassouni K, Youssoufi MA (2018) Clinical and Therapeutic Profile of Undifferentiated Nasopharyngeal Carcinoma in Children and Young Adults: A Retrospective Study. J Nucl Med Radiat Ther 9: 377. doi: $10.4172 / 2155-9619.1000377$

Page 3 of 4

\begin{tabular}{|l|l|}
\hline 3DCRT & $15(75)$ \\
\hline IMRT & $5(25)$ \\
\hline
\end{tabular}

Table 1: Patients characteristics.

\section{Response to induction chemotherapy}

Ten $(50 \%)$ patients were in complete remission, five (25\%) patients in partial remission, and no patients were progressing. However, 5 patients have not had a post-chemotherapy evaluation $(25 \%)$.

The toxicity secondary to chemotherapy was mainly haematological alone $(10 \%)$, gastrointestinal alone $(10 \%)$ and association of more than two toxicity in $15 \%$ of cases (haematological or gastrointestinal or renal).

\section{Response to radiotherapy}

Complete remission was described in 12 patients (60\%), partial remission in three patients (15\%) and two patients had loco-regional failure (at one year and two years). Distant metastasis occured in three patients (15\%).

In this study overall survival was $85 \%$ with $15 \%$ of deaths (3 patients). The causes of death included hemorrhagic shock secondary to pancytopenia, pulmonary embolism and unexplained cardiorespiratory arrest.

Disease free survival at 3 years was $50 \%$.

\section{Toxicity}

The acute and late toxicities for both 3DCRT and IMRT are summarized in Table 2.

\begin{tabular}{|c|c|c|}
\hline & $\begin{array}{l}\text { 3DCRT } \\
\text { Number (\%) }\end{array}$ & $\begin{array}{l}\text { IMRT } \\
\text { Number (\%) }\end{array}$ \\
\hline \multicolumn{3}{|l|}{ Acute Toxicity } \\
\hline Mucosa & $15(100)$ & $5(100)$ \\
\hline Skin & $9(60)$ & $3(60)$ \\
\hline \multicolumn{3}{|l|}{ Late Toxicity } \\
\hline Fibrosis & $8(53,3)$ & $3(60)$ \\
\hline Xerostomia & $11(73,3)$ & $2(40)$ \\
\hline Hearing impairement & $6(40)$ & $1(20)$ \\
\hline
\end{tabular}

Table 2: Acute and late toxicity after chermoradiotherapy.

\section{Discussion}

The incidence of nasopharyngeal cancer varies by region [6]. In endemic countries, the peak age is around the $5^{\text {th }}$ and $6^{\text {th }}$ decade, while in the Mediterranean basin, there is a second peak of age between 10 and 20 years with an incidence between $2 \%-18 \%$ [1].

The locally advanced stages are characteristic presentation of this form of cancer with a rate that varies between $30 \%$ and $92 \%$ according to the different pediatric series [7]. In our series, there was $75 \%$ of T3T4. This is probably due to the nonspecificity of the symptoms such as

nasal obstruction, epistaxis, hearing losses which are also present in benign conditions. The lack of access to care and diagnostic means in developing countries such as Morocco is also contributory.

Undifferentiated carcinoma of the nasopharynx is radiosensitive. Radiotherapy alone in children with advanced stage has a 5-year survival rate of $20 \%$ to $40 \%$ [8] The low survival and frequency of metastatic relapses in these stages led to the combination with chemotherapy according to different protocols (induction, concomitant, adjuvant) but still without standardized scheme given the small series of children [9].

The choice between concomitant or sequential radio chemotherapy has not yet been standardized due to the lack of randomized clinical trials [10]. In our series, patients received induction chemotherapy followed by concomitant chemo radiotherapy. This is due to the significant improvement of survival by this modality in randomized clinical trials in adults but also in a prospective Italian study in children [11-13].

Venkitaraman and colleagues in 2007, found that the radio chemotherapy combination was associated with a better three-year disease-free survival rate compared to exclusive radiotherapy (82\% vs. $40 \%, p=0.001$ ) [14]. Primary tumor volume is highly significant in evaluating local control, distant metastasis and overall survival [15]. In retrospective series, the radiotherapy dose greater than 66 Gy gave had a better disease free survival $[16,17]$. In our series all our patients had 70 Gy as a curative dose.

\section{Conclusion}

Most children and adolescents have locally advanced nasopharyngeal carcinoma. The radio chemotherapy has given good results. Reducing distant metastasis and late toxicity using new protocols and IMRT are future directions in the management of these cancers. Adaptive radiotherapy needs to be evaluated to minimize sequelae. Radiotherapy and chemotherapy protocols should be standardized through randomized, multicenter studies.

\section{Limitations}

Our serie is relatively small because of disease rarity in children even if Morocco is an intermediate endemic country like other northafrican ones. It was about 4 patients every year. The doses are $70 \mathrm{~Gy}$ with concomittant cisplatin for all patients unless the dose constraints to OAR such as brainstem couldn't be respected. This protocol was also reported in other series. Our doses are high because of the locally advance stages of our patients.

\section{Patient evaluation}

Actually, there was CR after chemotherapy in 10 patients and CR after radiotherapy in 12 patients. There was PR after chemotherapy in 5 patients and $\mathrm{PR}$ in three patients after radiotherapy (two of the initial 5 completed CR).

\section{According to WHO}

Complete remission is defined as total disappearence of any known lesion. Partial remission is defined as decrease of more than $50 \%$. Concerning the induction chemotherapy toxicity, unfortunately we only have data on whether it's hematological, gastrointestinal or renal. 
Citation: Erraisse MA, Mazghi AE, Bouhafa T, Hassouni K, Youssoufi MA (2018) Clinical and Therapeutic Profile of Undifferentiated Nasopharyngeal Carcinoma in Children and Young Adults: A Retrospective Study. J Nucl Med Radiat Ther 9: 377. doi: $10.4172 / 2155-9619.1000377$

Page 4 of 4

We don't have details because we assume that most of hematological are for example neutropenia.

\section{References}

1. Ayan I, Kaytan E, Ayan N (2003) Childhood nasopharyngeal carcinoma: From biology to treatment. Lancet Oncol 4: 13-21.

2. Daoud J, Toumi N, Bouaziz M, Ghorbel A, Jlidi R, et al. (2003) Nasopharyngeal carcinoma in childhood and adolescence: Analysis of a series of 32 patients treated with combined chemotherapy and radiotherapy. Eur J Cancer 39: 2349-2354.

3. Frikha M, Toumi N, Ghorbel L, Ben Salah H, Khabir A, et al. (2010) Le cancer du cavum de lenfant et l'adulte jeune : Aspects anatomocliniques, thérapeutiques et particularités évolutives. J canrad 14: 169-175.

4. Sultan I, Casanova M, Ferrari A, Rihani R, Rodriguez C (2010) Differential features of nasopharyngeal carcinoma in children and adults: A SEER study. Pediatr Blood Cancer 55: 279-284.

5. Downing NL, Wolden S, Wong P, Petrik DW, Hara W, et al. (2009) Comparison of treatment results between adult and juvenile nasopharyngeal carcinoma. Int J Radiat Oncol Biol Phys 75: 1064-1070.

6. Liu W, Tang Y, Gao L, Huang X, Luo J, et al. (2014) Nasopharyngeal carcinoma in children and adolescents-A single institution experience of 158 patients. Radiat Oncol 9: 274.

7. Laskar S, Sanghavi V, Muckaden MA, Ghosh S, Bhalla V, et al. (2004) Nasopharyngeal carcinoma in children: 10 years' experience at the Tata Memorial Hospital, Mumbai. Int J Radiat Oncol Biol Phys 58: 189-95.

8. Yi JL, Gao L, Huang XD (2006) Nasopharyngeal carcinoma treated by radical radiotherapy alone: Ten-year experience of a single institution. Int J Radiat Oncol Biol Phys 65: 161-168.

9. Nikitovic M, Popovic-Vukovic M, Stanic D, Bokun J, Paripovic L, et al. (2018) Treatment outcome of childhood nasopharyngeal carcinoma: A single institution experience. Int J Pediatr Otorhinolaryngol 113: 168-172.
10. Zrafi WS, Tebra S, Tbessi S, Ouni A, Jebsi M, et al. (2017) Undifferentiated carcinoma of nasopharyngeal type in children: Clinical features and outcome. Int J Pediatr Otorhinolaryngol 5: 321-324

11. Noël G, Dessard-Diana B, Vignot S (2002) Treatment of nasopharyngeal tumors: Literature review. Cancer Radiother 6: 59-84.

12. Casanova M, Bisogno G, Gandola L, Cecchetto G, Di Cataldo A, et al. (2012) A prospective protocol for nasopharyngeal carcinoma in children and adolescents. Cancer 118: 2718-2725.

13. Casanova M, Özyar E, Patte C, Orbach D, Ferrari A, et al. (2015) International randomized phase 2 study on the addition of docetaxel to the combination of cisplatin and 5-fluorouracil in the induction treatment for nasopharyngeal carcinoma in children and adolescents. Cancer Chemother Pharmacol 77: 289-298.

14. Venkitaraman R, Ramanan SG, Sagar TG (2007) Nasopharyngeal cancer of child- hood and adolescence: A single institution experience. Pediatr Hematol Oncol 24: 493-502.

15. Zaghloul MS, Eldebawy E, Ahmed S, Ammar H, Khalil E, et al. (2016) Does primary tumor volume predict the outcome of pediatric nasopharyngeal carcinoma: A prospective single-arm study using neoadjuvant chemotherapy and concomitant chemotherapy with intensity modulated radiotherapy. Asia Pac J Clinical Oncol 12: 143-150.

16. Laskar S, Sanghavi V, Muckaden MA, Ghosh S, Bhalla V, et al. (2004) Nasopharyngeal carcinoma in children: Ten years' experience at the tata memorial hospital, mumbai. Int J Radiat Oncol Biol Phys 58: 189-95.

17. Cheuk DK, Billups CA, Martin MG (2011) Prognostic factors and longterm outcomes of nasopharyngeal carcinoma in children and adolescents. Cancer 117: 197-206. 